\title{
Diacronie
}

Studi di Storia Contemporanea

$N^{\circ} 24,4 \mid 2015$

Le dittature militari: fisionomia ed eredità politica

\section{Nota introduttiva n. 24 - dicembre 2015}

Jacopo Bassi, Carlos Hudson e Matteo Tomasoni

\section{(2) OpenEdition}

\section{Journals}

\section{Edizione digitale}

URL: http://journals.openedition.org/diacronie/3578

DOI: 10.4000/diacronie.3578

ISSN: 2038-0925

\section{Editore}

Association culturelle Diacronie

\section{Notizia bibliografica digitale}

Jacopo Bassi, Carlos Hudson e Matteo Tomasoni, « Nota introduttiva n. 24 - dicembre 2015 »,

Diacronie [Online], № 24, 4 | 2015, Messo online il 29 décembre 2015, consultato il 23 septembre 2020.

URL : http://journals.openedition.org/diacronie/3578; DOI : https://doi.org/10.4000/diacronie.3578

Questo documento è stato generato automaticamente il 23 settembre 2020.

Creative Commons License 


\section{Nota introduttiva n. 24 - dicembre 2015}

Jacopo Bassi, Carlos Hudson e Matteo Tomasoni

Dittature militari, responsabilità politiche, interessi economici e vittime civili

Le dittature non sono soltanto pericolose, sono esse stesse sempre in pericolo poiché l'uso brutale della forza suscita ovunque ostilità ${ }^{1}$

1. Un concetto antico e un problema "filologico" 
Il termine dittatura nasce nel mondo romano - la tirannia greca non possiede le stesse caratteristiche legate all'ambito militare che ha a Roma - ed è una magistratura. Originariamente indicava una carica straordinaria a cui erano conferiti, in caso di pericoli immediati, poteri eccezionali, che facevano sì che tutte le altre magistrature fossero a lui

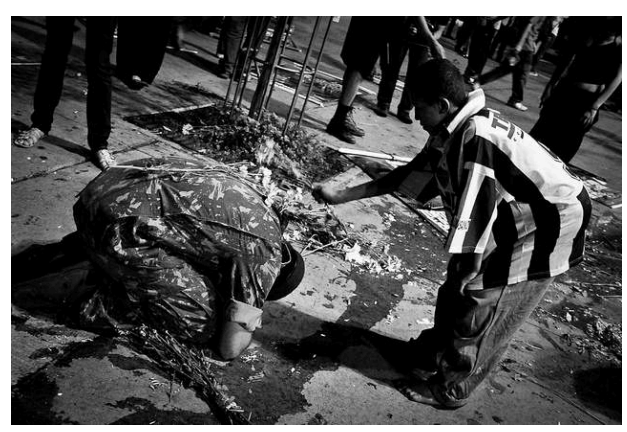
subordinate, anche se solo per la durata di sei mesi. Il termine dictator assunse però nel mondo romano, durante il corso dei secoli, accezioni e prerogative differenti. Se, dunque, «l'antica dittatura romana del tempo delle guerre sannitiche e delle lotte tra i patrizi e i plebei, che esigevano eguali diritti, era orientata in primo luogo a proteggere l'ordinamento esistente, minacciato da una situazione di emergenza, nell'interesse dell'aristocrazia, senza concedere al dittatore un incremento incontrollabile del suo potere [...] $»^{2}$ la prima dittatura "nuova", quella di Silla «si annoverava - per servirci di una formulazione di Mommsen - tra i poteri straordinari costituenti; rappresenta un potere monarchico pressoché illimitato, con lo scopo di creare un ordinamento nuovo per consolidare il dominio di una oligarchia, di un gruppo all'interno di una nobiltà» ${ }^{3}$. Alla prima dittatura di tipo "nuovo", quella di Silla, fece seguito quella di Cesare che covava in sé già le caratteristiche del futuro principato: all'abolizione dei limiti di tempo, si venne ad assommare, nel 44 a.C., l'abolizione dell'abdicazione, con il conferimento da parte del Senato del titolo di dictator perpetuus.

2 L'intervento straordinario di singoli individui per pacificare una situazione di disordine si verificò anche in epoca medioevale: $i$ casi dei podestà stranieri richiamati nei comuni italiani per porre un argine alla conflittualità tra i poteri locali, dei comandanti militari che conquistano il dominio su una città (Francesco Sforza su tutti) - mostrano la permanenza del modello di governante sostenuto dal potere militare per risolvere un momento politico difficile, caratterizzato dall'instabilità. Ma sono, soprattutto il segnale di un inscindibile legame fra il potere economico, civile, e quello militare, di chi detiene la forza ed è in grado di imporre l'ordine.

3 La distinzione fra i due tipi di dittature di epoca romana - evidenziata da Machiavelli, da Montesquieu, da Rousseau e dagli enciclopedisti - trovò la sua massima espressione durante la Rivoluzione francese, quando la discussione ruotò intorno al concesso di dittatura, in particolare in relazione alla figura di Robespierre ${ }^{4} \mathrm{e}$ al ruolo di dictator. Quella che per Rousseau doveva essere una magistratura temporanea ${ }^{5}$, assunse $i$ connotati di una carica vitalizia: Napoleone Bonaparte, in tal senso rappresentò con il colpo di Stato il fallimento dell'idea di dittatura temporanea. La rivoluzione, per difendere se stessa e i propri ideali avrebbe dovuto venir meno ai propri presupposti ideologici? Del resto il presupposto della condizione di transitorietà sarebbe riemerso anche nella formulazione del concetto di «dittatura del proletariato» da parte di Lenin ${ }^{6}$.

4 La condizione di transitorietà - connessa allo stato di eccezionalità - appare la ragione giustificante l'intervento del potere militare in ambito politico. 


\section{Lo stato di eccezionalità}

5 Proprio a partire dallo stato di eccezionalità i militari hanno giustificato il loro intervento in politica. Le dinamiche novecentesche non sono sfuggite a questa logica.

In termini generali, si può dire che - attraverso uno sguardo tecnico e astratto - il colpo di stato consista «nell'alterazione o distruzione dell'ordine politico da parte delle élite politiche o di determinati organismi dell'amministrazione, generalmente le Forze Armate, che ricorrono all'uso della violenza o alla minaccia con il fine di controllare o conquistare il potere statale» ${ }^{7}$. In altre parole, sarebbe «un cambio di governo effettuato da alcuni organismi in possesso del potere governativo la cui azione rappresenta una sfida alla legalità costituzionale dello Stato. [...] Il suo principale obiettivo è quello di alterare la politica statale attraverso un intervento a sorpresa e con il minor sforzo possibile» ${ }^{8}$.

7 Chi porta avanti il rovesciamento di un governo costituzionale deve, nonostante tutto, legittimare la propria azione perché tutto lo spazio politico, dopo il golpe, risulterà affetto dall'alterazione dei meccanismi di governo. A questo punto sorge un secondo elemento che si presenta nel momento in cui si analizza questo tema da un punto di vista teorico; esso ci conduce sul terreno della necessità in cui si sviluppa una motivazione concreta: «in qualsiasi caso, c'è sempre un riferimento che giustifica - agli occhi degli attori che vi prendono parte - una del tutto soggettiva "ragione di Stato" che spinge e motiva questi gruppi a compiere il golpe»". Questa situazione diventa imprescindibile, in quanto all'interno di questo processo, in qualsiasi settore dell'amministrazione in cui si collochi il normale funzionamento del 'gioco politico', la dialettica del consenso non si annulla, ed anzi, rimane attiva. Se l'uso o la minaccia della violenza - che in questo contesto corre il rischio di essere un monopolio - è lo strumento più importante per i golpisti, è nel campo della conquista del consensi dove esiste la possibilità di interazione tra gli attori che comporranno il nuovo scenario - che si gioca un'altra partita.

8 Rispetto a ciò che è stato appena detto, il timore dello scoppio di disordini sociali risulta onnipresente e la stessa possibilità che questi determinino una guerra civile può essere considerato come causa scatenante e giustificante l'intervento. Per risolvere questa ipotesi di conflitto, le Forze Armate, gruppo gerarchizzato e legato alla burocrazia statale, promuovono il colpo di stato. Portando avanti un'analisi astratta, osserveremo che non tutti gli agenti che vi prendono parte sono in grado di portare avanti un'azione come questa: «gli attori in grado di realizzare un colpo di stato, saranno le forze armate o quei gruppi governativi in grado di mobilitare determinati ambiti del potere, e cioè, più concretamente, alcuni membri delle amministrazioni statali in grado di sviluppare una tecnica che possa assicurare l'esito dell'azione» ${ }^{10}$. Per semplificare l'interpretazione teorica di questo fenomeno, lo si può riassumere in questi termini: «è un atto inaspettato, imminente, decisivo, potenzialmente violento ed illegale, la cui imprevedibilità risulta pericolosa sia per i congiurati, sia per le eventuali vittime, e che necessita di una grande precisione nella sua esecuzione. [...] I colpi di stato sono azioni relativamente segrete che ignorano o esulano dai "canali regolari" o delle regole del gioco quanto allo sviluppo. [...] Si tratta di una strategia illegale la cui volontà è quella di far cadere un governo, si basa su di una pianificazione segreta $\mathrm{e}$ molto attenta ai particolari, seguita da un attacco immediato al centro nevralgico dell'amministrazione attraverso la minaccia o l'uso della violenza da parte di un piccolo 
gruppo cospirativo presente all'interno dell'apparato statale. Un golpe si giudicherà "riuscito" quando riesce ad imporre un potere in un governo selezionato dai propri cospiratori» ${ }^{11}$.

9 Curzio Malaparte ha proposto un'interpretazione del golpe come un problema 'tecnico' ha fatto ricorso ad esempi europei relativi agli inizi del XX secolo. Citando Trotzkij, questi sostiene che «l'insurrezione non è un'arte, è una macchina. Per metterla in movimento c'è bisogno di tecnici, e solo dei tecnici potranno difenderla» ${ }^{12}$; osserviamo quindi come per Malaparte la questione centrale sia che il problema della conquista e difesa dello Stato non è un problema politico, ma un problema tecnico. Ed è proprio l'aspetto "tecnico" a costituire l'eredità e la linea di continuità fra i putsch militari del Novecento, in Europa e fuori dall'Europa.

10 Il fenomeno dei colpi di Stato militari diviene, successivamente alla Seconda guerra mondiale, una "prerogativa" dei paesi in via di sviluppo. 0 , per essere più precisi, sembra toccare gli Stati periferici (sia economicamente rispetto al "centro", sia geograficamente rispetto alle superpotenze).

11 Il caso spagnolo è celebre per essere stato, in quello che è conosciuto in Europa come il blocco occidentale, una delle più longeve dittature esistenti insieme a quella portoghese. Le cause che portarono all'inizio della guerra civile spagnola nell'anno 1936 sono note e senza dubbio riconducibili alla tensione esercitata dalle diverse forze politiche sorte durante gli inquieti anni della Seconda Repubblica. Il Generalísimo Francisco Franco iniziò a consolidare il suo potere sin dai primi mesi di guerra e ciò fu logicamente possibile grazie alla concentrazione di cariche statali nelle sue mani o in una ristrettissima cerchia di collaboratori a lui fidati. Non fu quindi difficile alla fine della guerra, quindi sin dal 1939, estendere tale dominio su tutta la nazione ed imporre un sistema autoritario che durò quasi quarant'anni. Le due colonne portanti del regime franchista furono l'Esercito e la Chiesa, le quali collaborarono attivamente alla configurazione del regime e della società, senza però interferire eccessivamente nei piani di un Caudillo che mantenne sempre sotto controllo anche queste istituzioni a cui era particolarmente legato. In questo caso Justine Guitard ci propone un'analisi che vuole approfondire il rapporto tra il potere autoritario di Franco e le organizzazioni a lui fedeli, cercando di individuare all'interno di esse le basi che favorirono anche la stabilità di questo regime.

Oltre alle due dittature della penisola iberica, i militari si impossessarono del potere anche in Grecia (1967-1974) - a questi casi si può aggiungere quello del colpo di Stato pilotato di Jaruzelski -, ma in questo caso possiamo riscontrare alcune differenze. Se i primi due casi risultano una permanenza dei totalitarismi precedenti alla Seconda guerra mondiale, quello greco si configura come frutto di una situazione esogena ${ }^{13}$ - il conflitto fra USA ed URSS, la Guerra fredda - che avrebbe toccato in misura ancora più rilevante i paesi dell'America Latina, l'Africa e l'Asia.

Proprio la pressione internazionale e la condizione di perifericità sono gli elementi alla base del caso della guerra dell'ogaden, scoppiata fra due governi guidati da militari: la Somalia e l'Etiopia. L'analisi di Francesco Maria Mengo evidenzia, però, come non si possa derubricare questo scontro a una guerra per procura fra USA e URSS sottolineando gli elementi di specificità dei due regimi. 


\section{La Dottrina della Sicurezza Nazionale, il consenso e il sostegno interno ai militari}

diversi settori della classe dirigente del paese unendoli questi ultimi alle Forze armate nella difesa del capitalismo. La sicurezza del paese sarebbe andata di pari passo con il suo sviluppo economico; tutto questo nella convinzione che un paese sottosviluppato sia vulnerabile e risulti più facile un avanzamento delle forze comuniste; per questa ragione «sarebbe stato necessario fissare una strategia che permettesse di conseguire una rapida crescita economica/industriale, generando in questo modo "attrattività" per la popolazione, evitando focolai di scontento, cooptazione da parte dei movimenti di opposizione e possibili rivolgimenti sociali» ${ }^{14}$.

A partire dallo studio dell'applicazione della Dottrina della Sicurezza nazionale, il saggio di Ivonne Barragán e Ana Belén Zapata ci propone un'analisi della repressione in Argentina. La persecuzione degli elementi che si opponevano al regime, è messa sotto esame nei casi di Bahía Blanca e Ensenada. In linea con questo studio si inserisce anche il discusso "caso di Monsignor Angelelli", icona del movimento di opposizione e simbolo della resistenza contro la politica e al Processo di Riorganizzazione Nazionale sorto poco dopo il golpe promosso dal generale Jorge Rafael Videla. L'indagine che ci presenta Mirko Giancola si propone di osservare fino a che punto le relazioni tra Stato e Chiesa determinarono la conformazione di un regime dittatoriale, la cui stabilità si prolungò tra il 1976 e il 1983 a conseguenza anche della dura repressione e alla continua violazione dei diritti umani esercitata dal potere militare.

Il professor Jose Alfredo Amaral Gurgel, autore di uno dei libri di riferimento per il regime militare brasiliano, Segurança e democracia ${ }^{15}$, affermava che lo Stato aveva diritto di disciplinare il funzionamento delle imprese tutelando il conflitto ed eliminando, ad esempio, ogni possibile conflittualità sociale. Ed è del controllo da parte del regime militare brasiliano sul mondo del lavoro, più esattamente sui sindacati, che si occupa il saggio di Alejandra Estevez e Marco Antônio Teixeira. La necessità di conseguire risultati economici giustificava, per i militari e per l'imprenditoria che sostenne il golpe, la soppressione delle libertà sindacali. In nome dello sviluppo concetto era consentito derogare alla democrazia o interpretare liberamente questo concetto.

17 I meccanismi che determinano il successo di un golpe o la successione all'interno delle dittature contano dunque sui legami delle potenti lobbies nazionali. Quando nel giugno del 1943 il giovane colonnello Juan Perón appoggiò il colpo di stato, questi venne beneficiato dal potere militare ascendendo in brevissimo tempo sino all'incarico di vice-presidente, anche se pagò a caro prezzo alcuni disaccordi con le alte cariche dello stato, finendo persino per essere incarcerato per breve tempo. Così come lo sintetizza Pascal Madonna, l'incontro con Eva Duarte prima e la pressione popolare poi, permisero a Perón non solo di riottenere la libertà, ma anche di raggiungere la nomina a Presidente nel 1946; il suo mandato durò dieci anni, durante i quali lo Stato argentino visse profondi cambiamenti ma anche un rapporto quasi del tutto inedito tra le alte sfere dello stato e la massa popolare.

Sulle strategie operate dai militari golpisti per costruire un consenso personale - un vero e proprio culto della personalità - si concentra il saggio di Claudio Mancuso, dedicato alle dittature militari africane nel contesto della decolonizzazione. 


\section{II ruolo dei media e il consenso}

19 La maggior parte dei contributi della sezione dedicata al Brasile analizza il ruolo dei media nel contesto dei governi militari. Eduardo Henrique Barbosa de Vasconcelos e Ana Lorym Soares si soffermano sulla storia del Movimento folclorista in Brasile e sui legami che si statuirono con il governo dei militari. La conservazione della tradizione e il consolidamento dell'identità nazionale erano un argomento che toccava l'interesse dei vertici dello Stato, così come dimostrato da Sonia Wanderley nel suo saggio sui rapporti tra il settore televisivo e la dittatura militare. Dobbiamo però prestare attenzione a non cadere nell'equivoco di generalizzare questo tipo di rapporto e trattare tutto il contesto senza operare i dovuti distinguo. Anche perché come sottolinea Asterito Lapera nella sua analisi di tre opere cinematografiche, l'atteggiamento del regime nei confronti del cinema e del mezzo televisivo non fu costante e andò incontro ad un'inevitabile apertura, consentendo la circolazione di idee che esprimevano una critica nei confronti delle politiche dello Stato.

Anche nel caso argentino il ruolo dei mass media fu molto importante per il sostegno dei regimi dittatoriali, specialmente attraverso la consuetudinaria pratica del consenso. Non mancarono però anche casi opposti, in cui la satira politica venne utilizzata come fondamentale arma di resistenza contro il regime; fu questo il caso della rivista «Tía Vicenta», che tra il giugno e luglio del 1966 si oppose tenacemente alla Revolución promossa dal generale Onganía. Partendo da queste basi, Bettina Favero e Francisco Mosiewicki ci propongono quindi un testo in cui si può osservare come ed attraverso quali canali si diffuse una satira politica in grado di deteriorare l'immagine del regime e mettere in pericolo una stabilità basata proprio sul consenso. Un'opposizione, se vogliamo, timida e limitata nel tempo, ma fondamentale per mantenere viva la resistenza al regime.

21 Il costante bisogno dei regimi di proiettare un'immagine positiva ma soprattutto funzionale del sistema sociale e politico instaurato con la dittatura, ha portato alla ricerca di varie soluzioni durante il corso della loro esistenza. Ariel Ignacio Slavutsky ci propone in questo caso uno studio teorico la cui metodologia parte dall'identificazione del regime dittatoriale con il Dispositivo Generador de Identidades (DGI). Il caso studiato, l'ultima dittatura argentina (conosciuta come Proceso de Reorganización Nacional), fu un sistema politico basato sul controllo dell'opinione pubblica ma anche sulla repressione attraverso il monopolio della violenza e la creazione di istituzioni educativo-culturali in grado di rafforzare e promuovere l'identità nazionale e quindi l'immagine della stessa dittatura. Un sistema che fu messo in pratica solo in parte, ma che ebbe comunque importanti risvolti nel processo di consolidamento di una dittatura che si prolungò per sette anni.

\section{Eredità e memoria}

22 Cosa rimane, dunque, a distanza di anni, dei colpi di Stato militari e dell'apparato repressivo installato da loro? Priscila Cabral Almeida, affronta il caso di Belo Horizonte e della questione della memoria del regime militare brasiliano che, inevitabilmente, tocca la città stessa e la definizione di se stessa. La creazione di un memoriale dedicato alle vittime del regime non è solo una modalità di compensazione, 
ma è un'operazione di scrittura della storia. Una storia che viene scritta direttamente attraverso i muri della capitale dello Stato del Minas Gerais.

La lenta ma graduale perdita di appoggio popolare dei regimi dittatoriali del Brasile (1985) e del Cile (1989), ebbe come principale risultato la richiesta di libere elezioni e l'avvento di un processo che trascinò questi paesi verso la transizione democratica. Tale opportunità fu il primo passo verso la normalizzazione delle strutture sociali, politiche ed economiche di queste nazioni, ma ciò non significò la scomparsa di personalità che avevano caratterizzato ed appoggiato l'avvento di un sistema di tipo autoritario. María Elena Besso Pianetto ci propone uno studio che cerca di approfondire proprio questi due casi, con l'idea di far luce sulle circostanze che portarono al cambio di regime, senza però dimenticare - è importante ribadirlo - la presenza di determinati elementi politici appartenenti al precedente sistema. In entrambi i casi, osserveremo come la classe dirigente fu eletta democraticamente dalla popolazione civile, ma non per questa ragione smise di delegare alcuni incarichi specifici ad elementi provenienti dal "vecchio" regime autoritario: singoli o lobbies politiche considerate comunque indispensabili per il passaggio dalla realtà autoritaria a quella democratica.

Il caso della dittatura cilena è ancora oggi oggetto di numerosi dibattiti. Le atrocità commesse dal regime di Augusto Pinochet sono - e continuano ad essere - una ferita aperta, nonostante i vari processi messi in atto dalle autorità giudiziarie nel corso degli ultimi anni. All'interno di questo numero, Nicolas Prognon ha cercato di indagare anche su questi fattori, "sezionando" le differenti fasi che portarono all'apertura e quindi alla transizione democratica di questo paese alla fine degli anni Ottanta. L'autore propone una revisione storica che fa luce sul maggior controllo sociale esercitato da Pinochet a partire dalla seconda metà degli anni Settanta, per poi indagare il processo di legalizzazione di un sistema basato sulla nuova Costituzione del 1980 e quindi il graduale smantellamento dell'apparato autoritario, favorendo quindi una lenta apertura verso quella "transizione pacifica" voluta dal sistema democratico. Questo processo è, come accennavamo prima, ancora oggi un elemento di studio e riflessione, spesso causa di forti contrasti tra nostalgici del regime ed oppositori dello stesso.

Nel complesso mosaico delle dittature sorte nei paesi latinoamericani, spicca il caso dell'Uruguay. Magdalena Schelotto propone una riflessione su un regime, come quello brasiliano, definito oggi "civile-militare" sorto dopo le elezioni del 1971 e coordinato dall'allora presidente Juan María Bordaberry. La dittatura fu in questo caso imposta a partire dall'anno 1973, quando le autorità competenti permisero ai militari di penetrare con la forza all'interno del sistema politico uruguaiano, impadronendosi poco a poco di buona parte delle organizzazioni politiche e sociali della nazione. Attraverso l'imposizione della Dottrina della Sicurezza Nazionale" basata sull'anticomunismo, il regime poté appropriarsi della maggior parte delle istituzioni e imporre un controllo organico sullo Stato che, sin dagli inizi degli anni Ottanta esercitò anche un forte controllo sul processo di transizione democratica. Fu per questo motivo che durante quell'epoca si produsse una controversia sul passaggio delle competenze politiche della nazione da un sistema all'altro, dimostrando come gli interessi militari si fossero estesi ben oltre le iniziali posizioni. Questa situazione ha dato origine a numerose questioni in sospeso che l'autrice cerca di ricongiungere all'interno della sua analisi. 

mettendo a disposizione degli autori i loro suggerimenti. Un sentito grazie a: Anna Bruzzone, Antônio Manoel Elíbio Jr., Esteban Elena González, Andreza Maynard, Dilton Cândido S. Maynard, Janaína Mello, Mariana Pozzoni, Karl Schurster.

Jacopo Bassi, Carlos Hudson e Matteo Tomasoni

\section{NOTE}

1. JÜNGER, Ernst, Trattato del Ribelle, Milano, Adelphi, 2012 [12ํㅡㄹ.], p. 31.

2. IRMSCHER, Johannes, La dittatura. Tentativo di una storia concettuale, in MELONI, Giovanni (a cura di), Dittatura degli antichi e dittatura dei moderni, Roma, Editori Riuniti, 1983, pp. 55-75, p. 59.

3. Ibidem.

4. Ibidem, pp. 66-67.

5. ROUSSEAU, Jean-Jacques, Il contratto sociale, 1. IV, § 6, Milano, BUR, 1995, pp. 177-181.

6. MELONI, Giovanni, Concetti romani e pensiero leniniano. A proposito di tribunato e dittatura, in ID. (a cura di), Dittatura degli antichi e dittatura dei moderni, Roma, Editori Riuniti, 1983, pp. 31-45.

7. DE ANDRÉS, Jesús, El voto de las armas. Golpes de estado en el sistema internacional a lo largo del siglo XX, Madrid, Catarata, 2000, p. 33.

8. GONZÁLEZ CALLEJA, Eduardo, Los golpes de Estado, Madrid, Arco Libros, 2003.

9. DE ANDRÉS, Jesús, El voto de las armas, cit., p. 33.

10. Ibidem.

11. GONZÁLEZ CALLEJA, Eduardo, Los golpes de Estado, cit.

12. MALAPARTE, Curzio, Tecnica del colpo di stato, in ID., Opere scelte, Milano, Mondadori, p. 209.

13. CONTOGEORGIS, Georges, Il fenomeno autoritario in Europa, in LASCHI, Giuliana (a cura di), Memoria d'Europa. Riflessioni su dittature, autoritarismo, bonapartismo e svolte democratiche, Milano, Franco Angeli, 2012, pp. 9-19.

14. VEIGA DOCKHORN, Gilvan, Quando a ordem é segurança e o progresso é desenvolvimento, 1964-1974, Porto Alegre, EDIPUCRS, 2002, p. 64.

15. GURGEL, Jose Alfredo Amaral, Segurança e democracia: uma reflexao politica, Rio de Janeiro, Olympio Editora, 1975.

\section{AUTORI}

\section{JACOPO BASSI}

Jacopo Bassi ha conseguito la laurea specialistica in Storia d'Europa presso l'Università degli Studi di Bologna. Nel corso dei suoi studi si è occupato di storia della Grecia e dell'Albania in età contemporanea e di storia e istituzioni della Chiesa ortodossa. Ha lavorato per le case editrici 
Éditions des femmes, Il Mulino e Zanichelli.

URL: < http://www.studistorici.com/2009/02/24/jacopo_bassi/ >

\section{CARLOS HUDSON}

Carlos Fernando Hudson è professore di storia presso la Facultad de Humanidades

dell'Universidad Nacional de Mar del Plata. Tra le altre pubblicazioni, ha curato, assieme a Rosalía Baltar, Figuraciones de siglo XIX. Libros, escenarios y miradas, Mar del Plata, Finisterre Editores, 2011. Attualmente è borsista del CONICET (Consejo Nacional de Investigaciones Científicas y Técnicas) e sta sviluppando un progetto dal titolo "El Gobierno de José María Guido. Problemas de historia política 1962-1963".

URL: < http://www.studistorici.com/2013/02/12/carlos-hudson/ >

\section{MATTEO TOMASONI}

Matteo Tomasoni (Trento, 1982) è Dottore in Storia Contemporanea. Ha dedicato buona parte delle sue ricerche allo studio del fascismo in Spagna ed ha discusso una tesi dedicata a uno dei padri fondatori del falangismo, Onésimo Redondo Ortega. Le sue ricerche si sviluppano intorno allo studio del fascismo transnazionale, la cultura politica dell'Europa tra le due guerre e l'uso della violenza come linguaggio politico. Si dedica anche allo studio della Prima Guerra Mondiale e da poco ha iniziato una collaborazione con il Museo Storico Italiano della Guerra di Rovereto. È membro della riviste Diacronie. Studi di Storia Contemporanea e Zibaldone. Estudios Italianos e fa parte del gruppo di ricercatori del fascismo (SidIF).

URL: < http://www.studistorici.com/2008/09/14/matteo-tomasoni/ > 